\title{
Standard Model Masses Explained
}

\author{
T. R. Mongan \\ Sausalito, CA, USA \\ Email: tmongan@gmail.co
}

How to cite this paper: Mongan, T.R. (2021) Standard Model Masses Explained. Journal of Modern Physics, 12, 983-987. https://doi.org/10.4236/jmp.2021.127060

Received: April 18, 2021

Accepted: May 22, 2021

Published: May 25, 2021

Copyright $\odot 2021$ by author(s) and Scientific Research Publishing Inc. This work is licensed under the Creative Commons Attribution International License (CC BY 4.0).

http://creativecommons.org/licenses/by/4.0/

\begin{abstract}
The Standard Model of particle physics requires nine lepton and quark masses as inputs, but does not incorporate neutrino masses required by neutrino oscillation observations. This analysis addresses these problems, explaining Standard Model particle masses by describing fundamental particles as solutions of Einstein's equations, with radii 1/4 their Compton wavelength and half of any charge on rotating particles located on the surface at each end of the axis of rotation. The analysis relates quark and lepton masses to electron charge and mass, and identifies neutrino masses consistent with neutrino oscillation observations.
\end{abstract}

\section{Keywords}

Standard Model Masses, Spherical Fundamental Particles, Einstein's Equations

\section{Introduction}

The particle physics Standard Model involves three trios of charged fermions, three neutrinos, three spin one bosons with average charge zero, and a scalar Higgs boson. The nine charged fermion masses must be provided as inputs to the Standard Model. Neutrinos are massless in the Standard Model, in conflict with observations of neutrino oscillations. This analysis resolves these problems by describing particles as solutions of Einstein's equations, with radii 1/4 their Compton wavelength $I$ and half of any charge on rotating particles located on the surface at each end of the axis of rotation, to explain Standard Model masses consistent with PDG 2020 [1] and neutrino mass error bars. Particle internal mass and pressure distribution is described by cubic equations for $l$ in each charge state, allowing at most three particles in each charge state. This analysis replaces the Standard Model assumption of massless neutrinos that conflicts with neutrino oscillation observations, and avoids point particle infinite density. Charge neutrality of the universe is important to the analysis. 


\section{Cubic Equations for Wavelengths from Mass and Pressure Distribution}

Internal particle mass and pressure distribution, involving volume $\sim l^{3}$, surface area $\sim l^{2}$, and diameter $l / 2$, results in cubic equations in $l$ and allows at most three particles in each charge state. Describing mass and pressure distribution with surface and linear elements requires minimum surface shell thicknesses and axial core radii near the Planck length $l_{P}=\sqrt{\frac{\hbar G}{c^{3}}}$. Total particle mass is the sum of mass equivalents of pressure, $\mathrm{m} / 2$, in the volume, mass equivalent of surface pressure $\frac{\pi}{4} S l^{2}$, and core mass $L l$. So $\frac{4}{3} \pi \rho\left(\frac{l}{4}\right)^{3}=\frac{4}{3} \pi \frac{\rho}{2}\left(\frac{l}{4}\right)^{3}+4 \pi S\left(\frac{l}{4}\right)^{2}+2 L\left(\frac{l}{2}\right)$. This cubic, written as $A l^{3}-B l^{2}-C l=0$ with $A=\frac{\pi}{96} \rho, B=\frac{\pi S}{4}$, and $C=2 L$, has discriminant $\Delta=B^{2} C^{2}-4 A C^{3}$.

\section{Charged Fermions}

The Standard Model involves three constituent quarks in protons. With electron charge $-e$ and two trios of quarks, hydrogen atom charge neutrality requires two charge $\frac{2 e}{3}$ up quark and one charge $\frac{-e}{3}$ down quark constituent in each proton. Using the fine structure constant $\frac{e^{2}}{\hbar c}=\frac{1}{137}$, electrostatic potential energy from repulsion between equal surface charges at opposite ends of the rotation axis is $\left(\frac{q e}{6}\right)^{2} /\left(\frac{l}{2}\right)=q^{2} m c^{2} \frac{e^{2}}{18 \hbar c}=q^{2} \frac{m c^{2}}{2466}$. With electron mass $m_{e}$ electrostatic potential energy of charged ground state fermions is identical if up quark mass $=4 m_{e}$ and down quark mass $=9 m_{e}$, well within PDG 2020 error bars.

Discriminant $\Delta$ is positive for fermions regardless of the sign of $B$, so the cubic has three real roots corresponding to three fermion Compton wavelengths in each charge state [2]. Wavelengths in each charge state are projections on the $I$ axis of vertices of Nickalls triangles, centered on average wavelength $l_{\text {avg }}(q)$ and circumscribed by circles with radius $R(q)$ intersecting the $l$ axis at $l_{\text {avg }}(q)+R(q)$. Largest wavelength $l_{1}(q)=l_{\text {avg }}(q)+R(q) \cos [\theta(q)]$, smallest wavelength $l_{3}(q)=l_{\text {avg }}(q)+R(q) \cos [\theta(q)+2 \pi / 3]$, and intermediate wavelength $l_{2}(q)=l_{\text {avg }}(q)+R(q) \cos [\theta(q)+4 \pi / 3]$. Nickalls triangle offset distance, in Figure 1 , is $d(q)=\sqrt{R^{2}(q)-D^{2}(q)}$, where $D(q)=R(q)-l_{1}(q)-l_{\text {avg }}(q)$. With up quark mass $=4 m_{e}$ and down quark mass $=9 m_{e}$ hydrogen atom charge neutrality relates lepton wavelengths to quark wavelength in charge states $\frac{2 e}{3}$ and $\frac{-e}{3}$ by $d(-e)=d\left(\frac{-e}{3}\right)-2 d\left(\frac{2 e}{3}\right)$. Strange quarks, with lowest mass and longest wavelength of $2^{\text {nd }}$ and $3^{\text {rd }}$ generation fermions, have greatest "leverage" 
on Nickalls triangle orientation, and PDG 2020 masses satisfy the $d(q)$ relation if strange quark mass is increased $0.5 \%$ to $93.45 \mathrm{MeV} / \mathrm{c}^{2}$.

Fermions described [3] as Godel solutions to Einstein's equations have matter energy density $\rho c^{2}=\frac{48 \hbar c}{\pi l^{4}}$, vacuum energy density $-\frac{1}{2} \rho c^{2}=-\frac{24 \hbar c}{\pi l^{4}}$, and fermion internal gravitational constants $G_{F i}=\frac{3 c^{3} l_{i}^{4}(q)}{\hbar\left[0.2 l_{i}(q)-l_{\text {avg }}(q)\right]^{2}}$. Fermion equatorial tangential velocity $>c$, allowing closed time-like curves, is pathological in Godel cosmologies but not a problem in fermions unchanged from creation to annihilation.

\section{Neutral Particles}

Considering our universe as a closed vacuum-dominated Friedmann solution of Einstein's equations so large it is almost flat, with Hubble constant $H_{0}=67.8 \mathrm{~km} \cdot \mathrm{sec}^{-1} \cdot \mathrm{Mpc}^{-1}$ and $\Omega_{\Lambda}=0.692$, cosmic vacuum energy density is $\rho_{v}=5.98 \times 10^{-30} \mathrm{~g} / \mathrm{cm}^{3}$ [4]. Electron neutrinos are neutrino ground state if electron neutrino vacuum energy density is the negative of cosmic vacuum energy density, with electron neutrino wavelength $l_{1}=\sqrt[4]{\frac{24 \hbar}{\pi \rho c}}$ and mass $m_{1}=1.36 \times 10^{-3} \mathrm{eV}$. Neutrino oscillation data [5] $m_{2}^{2}-m_{1}^{2}=7.37 \times 10^{-5}(\mathrm{eV})^{2}$ and $m_{3}^{2}-\frac{1}{2}\left(m_{1}^{2}+m_{2}^{2}\right)=2.50 \times 10^{-3}(\mathrm{eV})^{2}$ estimates $m_{2}=8.58 \times 10^{-3} \mathrm{eV}$, $m_{3}=5.08 \times 10^{-2}$ and neutrino mass sum $0.61 \mathrm{eV}$, about half Vagnozzi's [6] 0.12 $\mathrm{eV}$ upper bound on neutrino mass sum.

Electroweak symmetry breaking requires three vector bosons with zero average charge: $\mathrm{W}_{ \pm}$(mass $m_{w}=80379 \mathrm{MeV} / \mathrm{c}^{2}$ and $l_{w}=2.45 \times 10^{-3} \mathrm{~F}$ ) and $\mathrm{Z}$ (mass $m_{Z}=91187.6 \mathrm{MeV} / \mathrm{c}^{2}$ and $l_{z}=2.16 \times 10^{-3} \mathrm{~F}$ ). Two equal $\mathrm{W}_{ \pm}$wavelengths occur if discriminant $\Delta=0$, requiring $B^{2}=4 A C, \rho=\frac{3}{4} \pi \frac{S^{2}}{L}$, and vector boson internal gravitational constants $G_{B}=\frac{25 c^{3} l^{2}}{3 \hbar}$. Scalar Higgs bosons (mass $m_{H}=125100 \mathrm{MeV} / \mathrm{c}^{2}$ and $l_{H}=1.58 \times 10^{-3} \mathrm{~F}$ ) have no rotation axis, and no $l$ term in mass equivalent distribution. So only one charge zero scalar particle is allowed, $A l-B=0, S=\frac{2 \hbar c}{\pi l_{H}^{3}}$, and Higgs internal gravitational constant $G_{H}=\frac{c^{3} l_{H}^{2}}{12 \hbar}$.

These results account for the masses of all Standard Model particles with zero average charge.

\section{Relation to Point Particle Idealization}

Classical physics idealizes the force of gravity between extended bodies as the force between masses concentrated at the center of mass of each body. The ma- 
thematical construct corresponding to the center of mass of extended Standard Model particles provides a link to the idealization of point particles in quantum mechanics. At the instant of particle-antiparticle creation, the centers of mass of the respective Einstein solutions coincide and the spin axes are aligned. Correspondingly, at the instant of particle-antiparticle annihilation, the centers of mass of the respective Einstein solutions coincide and the spin axes are aligned.

\section{Conclusion}

Describing Standard Model particles as solutions of Einstein's equations, with radii $1 / 4$ their Compton wavelength and charge on rotating particles located on the surface at each end of the axis of rotation, explains Standard Model masses consistent with PDG 2020 and neutrino mass error bars. Study of interacting Einstein solutions with radii 1/4 Compton wavelength of their mass seems justified.

\section{Conflicts of Interest}

The author declares no conflicts of interest regarding the publication of this paper.

\section{References}

[1] Zyla, P.A., et al. (2020) (Particle Data Group 2020) Progress of Theoretical and EXperimental Physics, 2020, $083 \mathrm{C} 01$.

[2] Nickalls, R.W.D. (1993) The Mathematical Gazette, 77, 354. https://doi.org/10.2307/3619777

[3] Mongan, T.R. (2020) Journal of Modern Physics, 11, 1993-1998. https://doi.org/10.4236/jmp.2020.1112126

[4] Mongan, T.R. (2012) Holography, Large Scale Structure, Supermassive Black Holes and Minimum Stellar Mass. arXiv:1301.0304

[5] Capozzi, F. (2016) Nuclear Physics B, 908, 218. [arXiv:1601.07777].

[6] Vagnozzi, S. (2019) Cosmological Searches for the Neutrino Mass Scale and Mass Ordering. PhD Thesis, Stockholm University, Stockholm. [arXiv:1907.08010].

[7] Mongan, T. (2001) General Relativity and Gravitation, 33, 1415. https://doi.org/10.1023/A:1012065826750

[8] Bousso, R. (2002) Reviews of Modern Physics, 74, 825. [arXiv:hep-th/0203101]. https://doi.org/10.1103/RevModPhys.74.825

[9] Mongan, T.R. (2021) Journal of Modern Physics, 12, 295-299. https://doi.org/10.4236/jmp.2021.123022 


\section{Appendix: Cosmological Context}

Our universe can be described as a closed vacuum-dominated Friedmann solution of Einstein's equations so large it is almost flat. If there are compact extra dimensions, the universe could originate by quantum fluctuation from nothing [7], with dark energy identified as energy associated with compact dimensions. The holographic principle [8] indicates only $N=\frac{3}{8 G \rho_{v}}\left(\frac{c}{l_{P}}\right)^{2}=4.69 \times 10^{122}$ bits of information will ever be available to describe the observable universe. With no source or sink for information outside a closed universe, the number of bits of information in the universe remains constant. Two states $\pm \frac{e}{6}$ of each bit define electric charge $e$, and origin of the universe by quantum fluctuation from nothing requires zero net charge in the universe. Energy difference between bit states requires a small antimatter/matter ratio, consistent with observations [9].

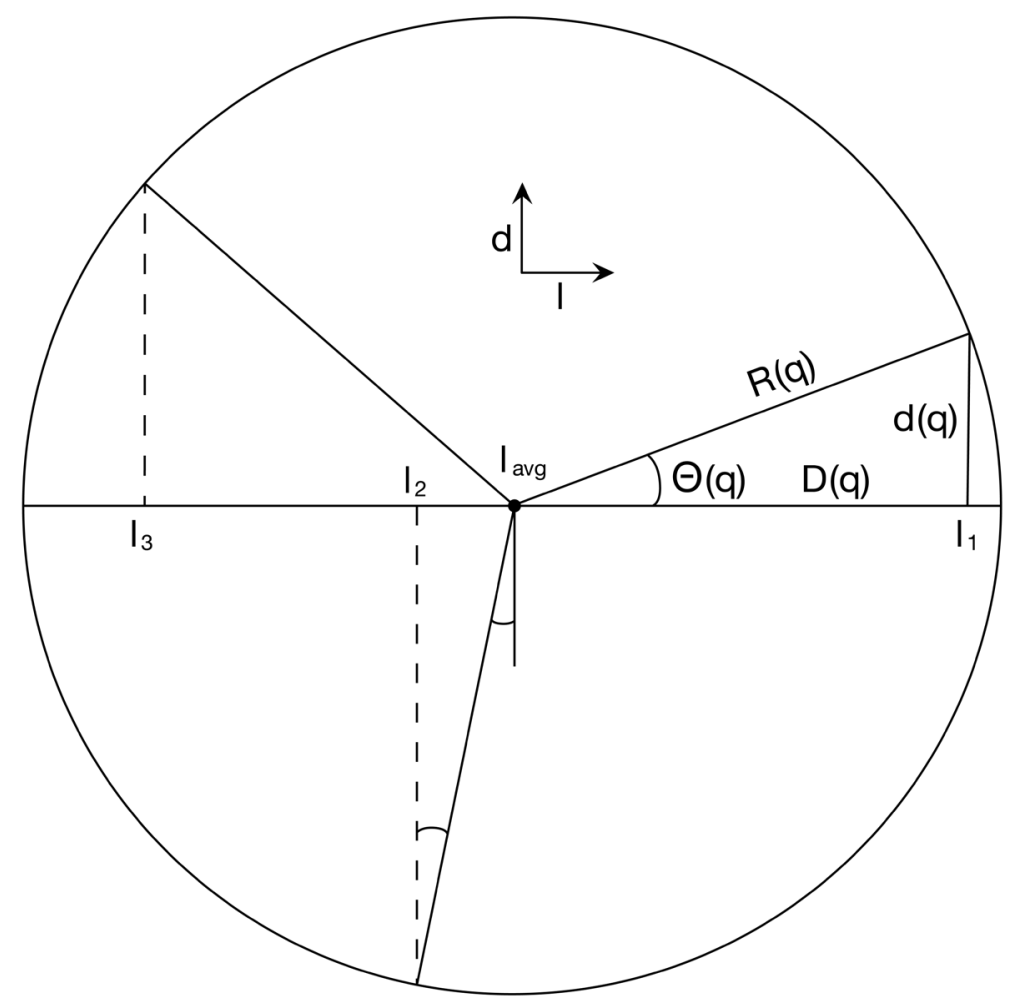

Figure 1. Particle wavelengths related to Nickalls triangle vertices on circumscribing circle. 